\title{
Self-management of salt intake: clinical significance of urinary salt excretion estimated using a self-monitoring device
}

\author{
Kenichiro Yasutake ${ }^{1}$, Noriko Horita ${ }^{2}$, Yoko Umeki ${ }^{2}$, Yukiko Misumi ${ }^{2}$, Yusuke Murata ${ }^{3}$, Tomomi Kajiyama ${ }^{1}$, \\ Itsuro Ogimoto ${ }^{1}$, Takuya Tsuchihashi ${ }^{4}$ and Munechika Enjoji ${ }^{3}$
}

Self-measured salt excretion from overnight urine samples shows significant correlation with 24-h-urinary salt excretion, but it is not known whether a self-measuring method can monitor daily fluctuations in individual salt consumption. In this study, we measured salt excretion from 24-h urine samples (24-h salt) in 50 volunteers over 3 test days ( 2 weekdays and 1 holiday), and examined to what extent the values correlated with estimates of 24-h salt excretion from overnight urine samples obtained using a self-monitoring device (ON salt). Urine collection was considered successful when the difference between the predicted and actual 24-h-urinary creatinine excretion was within $30 \%$. Thirty-three $(M / F=7 / 26 ; 39.6 \pm 16.7$ years) out of 50 participants completed their urine collections successfully and their samples were used in the analysis. Twenty-four-hour salt and ON salt did not significantly differ between test days and between the weekdays and the holiday. Moreover, there was a significant positive correlation between 24-h salt and ON salt for each test day. The coefficients of variation (CVs) for 24-h salt among test days and among subjects were $24.7 \%$ and $21.3 \%$, respectively. The CVs for ON salt were lower than those for $24-\mathrm{h}$ salt $(13.3 \%$ and $17.7 \%$, respectively). In conclusion, self-measurement of salt excretion from overnight urine samples allows estimation of daily salt intake; thus, the use of a self-monitoring device may be a useful motivational tool for personal salt restriction.

Hypertension Research (2016) 39, 127-132; doi:10.1038/hr.2015.121; published online 12 November 2015

Keywords: motivational tool; salt intake; self-monitoring device; salt restriction; urinary salt excretion

\section{INTRODUCTION}

Over $25 \%$ of deaths among Japanese people are attributed to cardiovascular disease, the main causes of which are hypertension and overconsumption of salt. ${ }^{1,2}$ The Japanese are known to consume more salt than many other nationalities; ${ }^{3-5}$ therefore, reduction of salt consumption is important for decreasing blood pressure and preventing the onset of cardiovascular disease. ${ }^{6,7}$ According to the I-Change Model 2.0 by de Vries et al., both awareness of daily salt consumption and motivation to continue salt reduction are important to encourage the prevention/improvement of excessive salt intake. ${ }^{8,9}$ However, it is hard to understand individual daily salt consumption because daily salt intake varies widely with dietary preferences. ${ }^{10,11}$

To evaluate individual salt intake, a method combining both accuracy and simplicity is ideal; however, dietary salt intake is one of the most difficult nutrient intakes to measure precisely. In practice, to obtain accurate results requires a complicated measurement method. When the emphasis is on simplicity, the accuracy of the measurement deteriorates. The most reliable results may be obtained by measuring excreted salt in urine samples collected over $24 \mathrm{~h}$, because about $98 \%$ of salt intake is absorbed and $86 \%$ of that is excreted into the urine. ${ }^{12}$ Although a 24-h-urine collection method has been examined in some hypertension-specific hospitals, the technique is difficult in practice because it imposes a large burden on the tested individuals. ${ }^{13,14}$ In addition, the values obtained from urine collected over a 24-h period do not reflect long-term salt intake. ${ }^{15,16}$ The use of diet history questionnaires, conducted with assistance from a registered dietitian, is a popular method for evaluating salt intake. However, the results obtained from the questionnaire depend on the memory and adherence of the subjects, and the techniques used by the interviewer. ${ }^{17}$ Moreover, the questionnaire is also inconvenient for the subjects to repeat. Another method used to assess salt intake is the spot urine or second-morning urine test, which is easier for subjects. ${ }^{18-20}$ However, this method has poorer reliability than 24 -h-urine collection, ${ }^{21}$ and creatinine and sodium must be measured in a laboratory. Although estimation of 24-h-urinary salt excretion from overnight urine samples using a

${ }^{1}$ Department of Nutritional Sciences, Faculty of Nutritional Sciences, Nakamura Gakuen University, Fukuoka, Japan; ${ }^{2}$ Department of Health and Nutrition Sciences, Faculty of Health and Nutrition Sciences, Nishikyushu University, Saga, Japan; ${ }^{3}$ Health Care Center, Fukuoka University, Fukuoka, Japan and ${ }^{4}$ Hypertension Center, Steel Memorial Yawata Hospital, Kitakyushu, Japan

Correspondence: Dr K Yasutake, Department of Nutritional Sciences, Faculty of Nutritional Sciences, Nakamura Gakuen University, 5-7-1 Befu, Jonan-ku, Fukuoka 814-0198, Japan.

E-mail: yasutake@nakamura-u.ac.jp

Received 16 March 2015; revised 7 August 2015; accepted 9 September 2015; published online 12 November 2015 
self-monitoring device is commonly considered to be inaccurate, ${ }^{21}$ results obtained using this method show significant correlations with salt excretion levels measured by the 24-h-urine collection method. ${ }^{22}$ Use of a self-monitoring device is expedient for personal use and repeated measurements; ${ }^{23}$ thus, the self-monitoring device is one of the few methods available for conveniently estimating daily fluctuations in salt intake. ${ }^{10,11}$ The use of a self-monitoring device motivates its users to continue sodium restriction, resulting in prevention of hypertension and cardiovascular diseases.

Accordingly, it is important to clarify whether daily changes in salt intake can be accurately estimated using a self-monitoring device. In this study, 24-h-salt excretion values were compared with estimates obtained from overnight urine samples using a self-monitoring device.

\section{METHODS}

\section{Subjects}

Fifty volunteers (aged 20-70 years, male/female $=10 / 40$ ) living in Saga or Fukuoka Prefecture, Japan, participated in this study. The exclusion criteria included subjects with physical, psychological and social difficulties; those without the ability for self-selection and/or self-determination; those undergoing medical treatment with diuretics; or those with edema. The study protocol adhered to the ethical guidelines of the 1975 Declaration of Helsinki and was approved by the Ethics Committee of Nishikyushu University. The subjects were given oral and written explanations of the purpose and procedures of the study, and written informed consent was obtained from all the subjects before participation.

\section{Intervention schedule}

All subjects were registered within February 2014, and 24-h-urine collection was performed over 3 days (contiguous or noncontiguous) during March and April 2014. The 24-h urine samples were then used to measure 24-h-urinary salt excretion. Given the possibility that salt intake might vary between weekdays and holidays, the 3 test days comprised 2 weekdays and a holiday. In subjects with irregular working hours, a holiday was defined as a nonworking day. In the case of female volunteers, non-menstrual days were selected. Sub-samples of 8 -h overnight urine were taken from the 24 -h urine samples and used to estimate of 24-h-urinary salt excretion with a self-monitoring device. During the study period, subjects were allowed to take routine meals and no dietary restrictions were imposed.

\section{Twenty-four-hour urine collection}

Twenty-four-hour urine was collected by a proportional sampling method using a partition cup, which proportionally collects $1 / 50$ of the total 24 -h urine volume. ${ }^{24}$ Urine volume and excreted urinary salt estimated for a 24 -h period using the proportional sampling method have been shown to be highly correlated with direct measurements of the same parameters from total 24-h

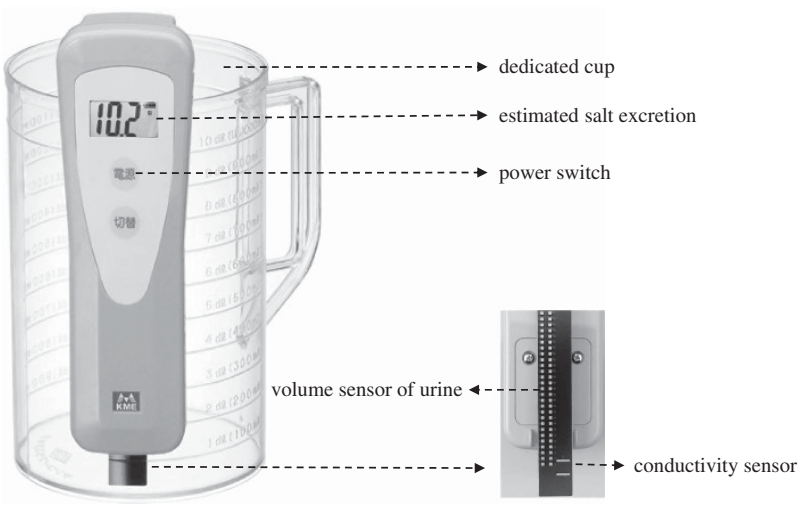

Figure 1 The self-monitoring device. A full color version of this figure is available at Hypertension Research journal online. urine, with the correlation coefficients reported as 0.97 and 0.98 , respectively. ${ }^{24}$ To ensure accurate use of the partition cup, oral and written explanations were provided and practice urine sampling was performed in advance of the actual collection. The subjects were obligated to collect a certain amount of urine while defecating.

Twenty-four-hour urine was defined as the total volume of urine starting with the second-morning urine (after discarding the first one at 0600 hours) and ending with the first-morning urine of the next day. Eight-hour overnight urine was defined as the urine excreted between 2200 hours (before sleep) and 0600 hours (the first-morning urine), which was collected in the selfmonitoring device and, after the excreted salt was measured, added back into the partition cup for 24-h collection. The precise start and end times for the urine collections were reported by each subject. Urine volumes and urinary creatinine, sodium and potassium concentrations were determined by the SRL Corporation (Tokyo, Japan). Creatinine was measured using an enzymatic method and sodium and potassium were measured using an electrode method. Urine collection was considered to be successful when the difference between the predicted and actual values for 24-h-urinary creatinine excretion was within $30 \% \cdot 13,14,25$ Subjects for whom any samples were unsuccessfully collected were excluded from the study. Urine samples were examined at any time after collection. The coefficient of variation (CV) of excreted salt (\%) was calculated using the following formula: (s.d./mean value) $\times 100$.

\section{Self-monitoring device}

The KME-03 self-monitoring device (Kohno ME Laboratory, Kanagawa, Japan) was used in this study (Figure 1). This device is capable of estimating 24-h-salt excretion from 8-h-overnight urine samples collected in an exclusive container. The subjects were asked to urinate $8 \mathrm{~h}$ before a prearranged wake-up time (0600 hours). The first urination after the sleeping period was collected in a dedicated cup. If unscheduled urination was needed between 2200 hours and 0600 hours, this urine was added to the first-morning urine and analyzed.

The self-measuring device contains a volume sensor and a conductivity sensor. The volume sensor consists of 50 small resistant chips, and the conductivity sensor consists of two gold-plated nickel metal plates. These sensors measure urine volume, urinary chloride concentration and temperature. These values are then integrated and the sodium chloride $(\mathrm{NaCl})$ concentration is estimated using an original algorithm. The $\mathrm{NaCl}$ concentration was adjusted using a correlation formula so that it was between the value obtained with the ion electrode method and the value for the conductivity method, because measurement of concentration by conductivity is affected by other electrolytes such as potassium. The self-monitoring device estimates 24-h-salt excretion from overnight urine samples using the following formula: $Y=5.76(X)^{0.53}$ g per day, where $Y$ is the estimated 24-h-urinary salt excretion and $X$ is the sodium content of the overnight urine samples. ${ }^{22}$ The error of each measurement by the self-monitoring device was defined as the estimated 24-h-urinary salt excretion from the 8 -h overnight urine samples (ON salt) minus the measured excreted salt from 24-h-urine samples (24-h salt).

\section{Statistical analysis}

Data analysis was performed using JMP version 10.0 software (SAS Institute, Cary, NC, USA). Measured data are presented as means \pm s.d. Between-group differences were assessed using a paired $t$-tests. Correlations were evaluated using a Pearson product-moment correlation coefficient. The sensitivity and specificity of the self-monitoring device for estimation of 24-h urinary salt excretion from overnight urine samples were assessed by logistic regression analysis and receiver operating characteristic curves. Differences were considered statistically significant if $P<0.05$.

\section{RESULTS}

Twenty-four-hour urine samples were collected by all 50 participants. Thirty-three of the 50 participants completed their urine collections successfully and their samples (sample number: $33 \times 3$ days $=99$ ) were used in the analysis. Among the excluded subjects, 12 (24\%) successfully collected for 2 days, four (8\%) for 1 day and one (2\%) could not collect any samples successfully. The percentages of subjects 
who successfully collected their urine samples were $88 \%(44 / 50)$ on the first weekday, $88 \%(44 / 50)$ on the second weekday and $78 \%$ (39/50) on the holiday. Overnight urine collection between 2200 hours. and 0600 hours was successfully completed in all participants and the urine samples were supplied for measurement by the self-monitoring device. The background characteristics of the 33 subjects whose samples were analyzed are shown in Table 1. Data from two hypertensive subjects who were taking non-diuretic antihypertensive agents were included in the analysis. Their mean blood pressure was within the normal range and their mean 24-h urinary $\mathrm{NaCl}$ and potassium excretion over 3 days were as follows: $\mathrm{NaCl}$ ( $24 \mathrm{~h}$ salt) $8.3 \pm 2.6$ g per day; potassium $1749 \pm 518 \mathrm{mg}$ per day; and $\mathrm{Na} / \mathrm{K}$ ratio $3.4 \pm 1.4$ (Table 2). The mean ON salt for all subjects over the 3 days was $8.1 \pm 1.8 \mathrm{~g}$ per day (Table 2 ). The mean 24 -h salt and $\mathrm{ON}$ salt

Table 1 Characteristics of participants

\begin{tabular}{lc}
\hline Number & 33 \\
\hline Sex: male/female & $7 / 26$ \\
Age (years) & $39.6 \pm 16.7$ \\
Body mass index $\left(\mathrm{kg} \mathrm{m}^{-2}\right)$ & $23.1 \pm 3.4$ \\
Systolic blood pressure (mm Hg) & $118.3 \pm 15.3$ \\
Diastolic blood pressure (mm Hg) & $73.2 \pm 10.8$
\end{tabular}

Values are means \pm s.d. values were $8.0 \pm 1.8$ and $8.0 \pm 1.7 \mathrm{~g}$ per day on day 1 (weekday), $8.5 \pm 2.6$ and $8.1 \pm 1.8 \mathrm{~g}$ per day on day 2 (weekday), and $8.3 \pm 3.3$ and $8.3 \pm 2.0 \mathrm{~g}$ per day on day 3 (holiday), respectively. No significant difference was found between 24-h salt and $\mathrm{ON}$ salt, or between weekdays and holidays (Table 2). Moreover, a significant positive correlation was observed between 24 -h salt and $\mathrm{ON}$ salt on day 1 $(r=0.44, P=0.01)$, day $2(r=0.40, P=0.02)$ and day $3(r=0.44$, $P=0.01$; Figure 2). There was no correlation between blood pressure and $\mathrm{ON}$ salt or 24-h salt on any day.

When the measurement errors between 24-h salt and ON salt were assessed, overestimation or underestimation was shown in some subjects (Table 3). The CVs for 24-h salt among test days and among subjects were $24.7 \%$ and $21.3 \%$, respectively. The CVs for ON salt were lower than those for 24 -h salt $(13.3 \%$ and $17.7 \%$, respectively; Table 2). Analysis of the correlation between 24-h salt and the measurement errors (ON salt minus 24-h salt) revealed a significant negative correlation $(r=-0.76, P<0.0001$; Figure 3$)$. The slope of the regression line was $<1.0$, and there was a trend toward overestimation and underestimation in the lower and higher ranges of the 24-h salt values, respectively.

When achievement of the target salt intake $(7 \mathrm{~g}$ per day, adopted from the Overview of Dietary Reference Intakes for Japanese $2015^{26}$ was defined as an objective variable and ON salt was defined as an explanatory valuable, logistic regression analysis revealed a significant association between the two values $(P<0.005$, odds ratio 0.63$)$. In the

Table 2 Measured urine sample parameters

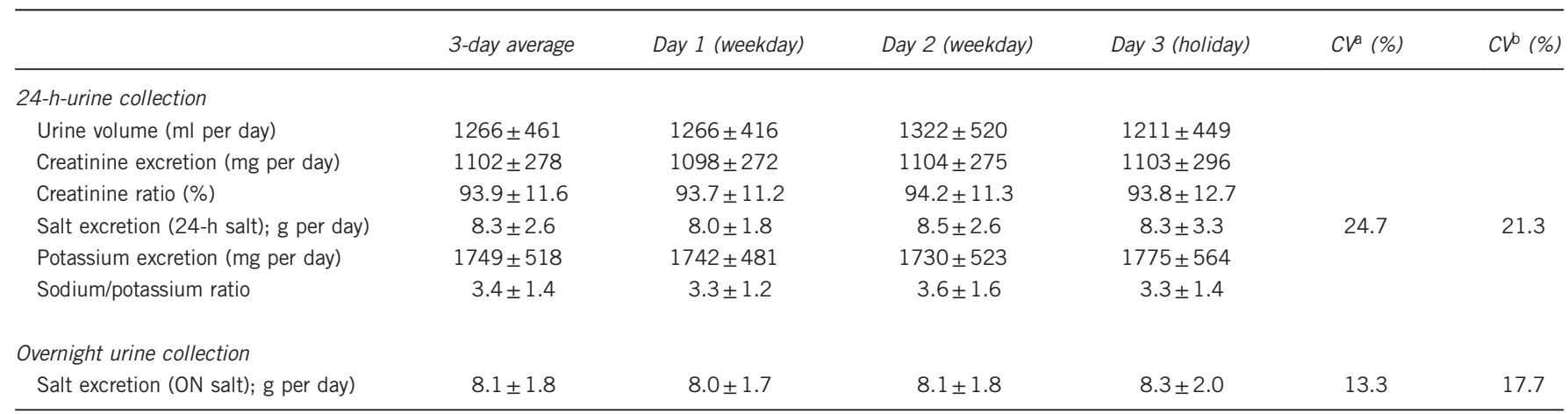

Abbreviation: $\mathrm{CV}$, coefficients of variation.

The ratio of observed to expected creatinine excretion calculated using the equations of Kawasaki et al. ${ }^{15}$

${ }^{\mathrm{a}} \mathrm{CV}$ among test days.

${ }^{\mathrm{b}} \mathrm{CV}$ among subjects.

a

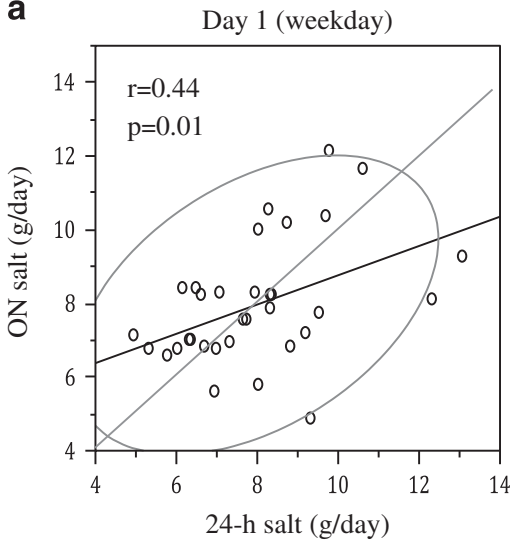

b

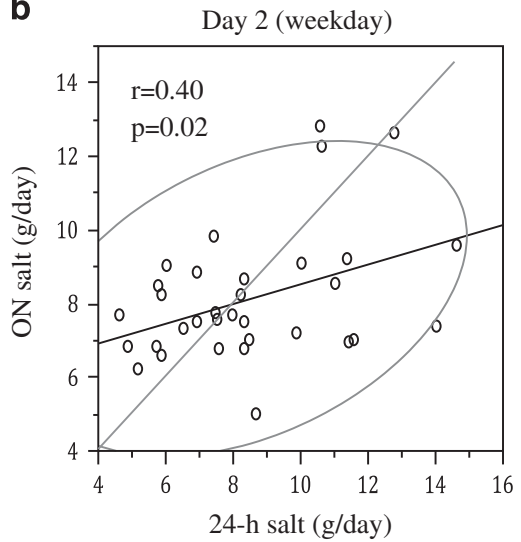

c

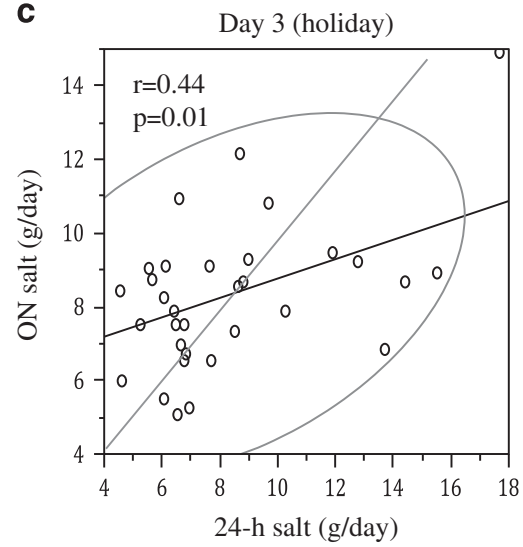

Figure 2 Correlation between salt excretion from 24-h urine samples and from overnight urine samples. (a) Day 1 (weekday), (b) Day 2 (weekday), (c) Day 3 (holiday). ON salt, salt excretion from overnight urine samples estimated using a self-monitoring device; 24-h salt, salt excretion from 24-h urine samples. 
Table 3 Measurement errors between 24-h salt and ON salt

\begin{tabular}{lccc}
\hline & \multicolumn{3}{c}{ ON salt minus 24-h salt (g per day) } \\
\cline { 2 - 4 } & $\begin{array}{c}\text { Day 1 } \\
\text { (weekday) }\end{array}$ & $\begin{array}{c}\text { Day 2 } \\
\text { (weekday) }\end{array}$ & $\begin{array}{c}\text { Day 3 } \\
\text { (holiday) }\end{array}$ \\
\hline Maintiles & 2.3 & 3.0 & 6.3 \\
75th & 1.4 & 1.8 & 2.2 \\
50th & 0.3 & 0 & 0.2 \\
25th & -1.0 & -1.9 & -1.6 \\
Minimum (underestimation) & -4.5 & -6.7 & -6.9 \\
Mean \pm s.d. & $0 \pm 1.9$ & $-0.4 \pm 2.5$ & $0 \pm 3.1$ \\
\hline
\end{tabular}

ON salt, salt excretion from overnight urine samples; 24-h salt, salt excretion from 24-h urine samples.

receiver operating characteristic analysis, the cut off value for ON salt that correlated with a value of $>7 \mathrm{~g}$ of 24 -h salt was $7.6 \mathrm{~g}$ (sensitivity 0.71 , specificity 0.32 ; Figure 4 ).

\section{DISCUSSION}

In this study, whether ON salt (self-monitoring device method) could properly reflect 24 -h salt (24-h urine collection method) was assessed in enrolled volunteers over 3 test days. Although all 50 participants provided 24-h urine samples, the percentage of subjects who successfully completed all 3 days of urine collection (including weekdays and holidays) was only $66 \%$ (33/50); this suggests that, especially for 24 -h urine collection, it is difficult to achieve successful urine collection on multiple test days. Although the self-monitoring device method usually requires single urine collection and there is little possibility of failure, in this study all participants successfully completed the overnight 8-h urine collection. Namely, since the success rate is not satisfied in the 24-h urine collection method, the self-monitoring device method is worthwhile for estimation of 24-h urinary salt excretion.

It has been reported that 24 -h urinary salt excretion has a definite association with self-measured salt excretion from overnight urine samples. ${ }^{22}$ In our previous salt intake and excretion study using a selfmonitoring device and test meals containing known amounts of salt $(5,10$ and $13 \mathrm{~g}$ per day of salt), we confirmed that $\mathrm{ON}$ salt roughly reflected the previous day's salt intake, ${ }^{25,27}$ and that, in the case of $10 \mathrm{~g}$ per day salt intake, there was a significant correlation between $\mathrm{ON}$ salt and 24 -h salt. ${ }^{25}$ However, these results have not been tested in the same subjects over multiple days. The novelty and significance of this study were that, in the daily life of 33 subjects, 24-h salt and ON salt were simultaneously assessed and compared over 3 test days and on each test day there was a significant correlation between them. These findings indicate that a self-monitoring device can estimate salt intake, which fluctuates daily, with sufficient accuracy to have real-life application. However, the actual salt intake/excretion of each subject cannot be estimated using the measured values obtained from the self-monitoring device because the correlation coefficient $(\approx 0.4)$ was not high enough to allow this. In practice, the maximum measurement errors between 24-h salt and ON salt were more than $6 \mathrm{~g}$ per day (Table 3 ). The absolute mean value of the errors was quite small when 24-h salt ranged from 6 to $9 \mathrm{~g}$ per day, but was larger at lower or higher levels of 24-h salt (Figure 3).

It has previously been shown that the self-monitoring device method overestimated 24-h salt within the lower range of salt excretion. ${ }^{22}$ In addition, in our salt intake and excretion study, the self-monitoring device method over- and under-estimated 24 -h salt in

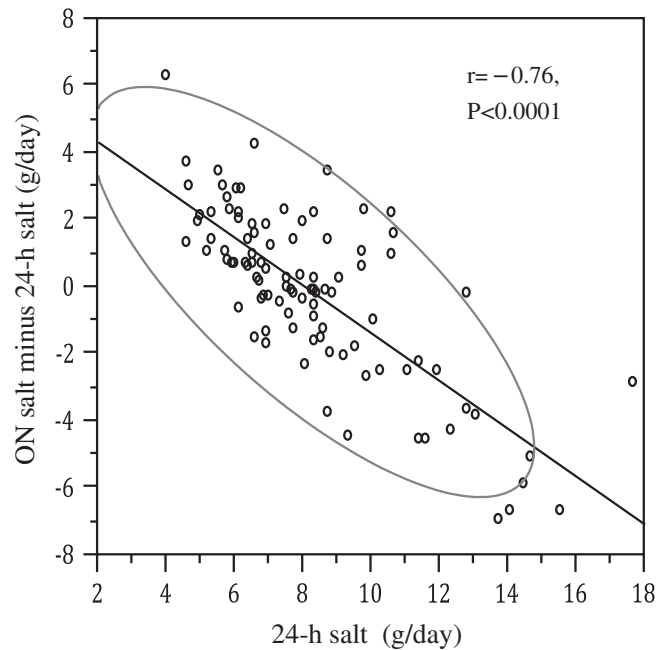

Figure 3 Relationship between salt excretion from 24-h urine samples and measurement errors. Correlation between salt excretion from 24-h urine samples (24-h salt) and measurement errors (ON salt minus 24-h salt).

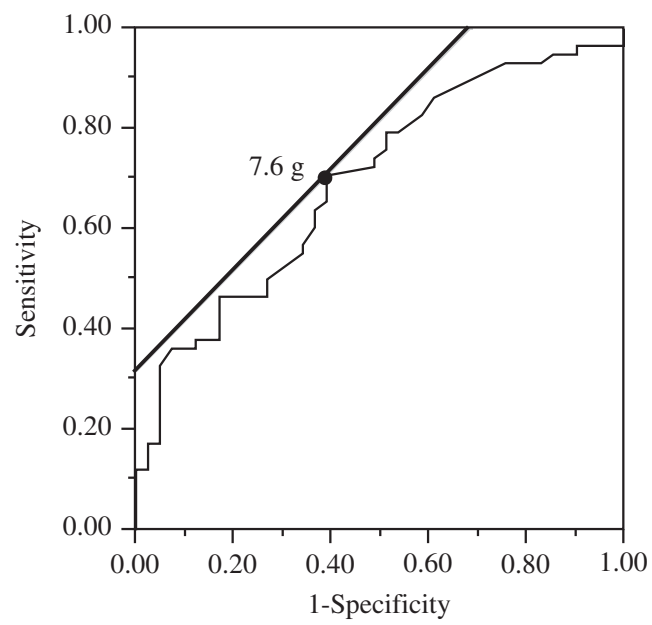

Figure 4 Receiver operating curve: sensitivity and specificity of 24-h salt excretion from overnight urine samples.

the lower and higher ranges of salt intake/excretion, respectively. ${ }^{25,27}$ These results confirm the validity of the results of our current study. The CVs for 24-h salt among test days and among subjects coincided approximately with those in a previous report that investigated salt excretion in healthy Japanese adults, ${ }^{26}$ whereas the CVs for ON salt were much lower than those for 24 -h salt (Table 2). These observations suggest that the suitable measurement range for the self-monitoring device is narrow and this may lead to over- and under-estimation relative to actual values when using the selfmonitoring device method, which may limit its effective measurement range. Thus, the device should only be employed with sufficient understanding of its characteristics. Conceivable causes of the measurement errors in the self-monitoring device method are as follows. Using a 24-h urine collection method, urine volume and urinary creatinine, sodium and potassium concentrations are measured directly. In the self-monitoring device method, volume and conductivity sensors measure urine volume, urinary chloride concentration, and temperature and 24-h salt is estimated using an original algorithm. Because the self-monitoring device measures 
urinary chloride concentration but not sodium concentration, increased potassium intake leads to overestimation of salt excretion. Possible causes of under- or over-estimation of salt excretion include impaired daytime salt excretion with compensatory nighttime excretion in participants with salt sensitivity, ${ }^{23,24}$ and individual differences in sodium excretion during the day or night leading to errors in the estimation of daily salt consumption using overnight urine samples. However, it was impossible to know if the subjects in this study had salt sensitivity.

Therefore, the self-monitoring device may be inappropriate to the instruction of patients with high salt intake. We consider the self-monitoring device method as a tool for detecting patients with high salt intake. Namely, subjects in whom estimated urinary salt was continuously high $(>8 \mathrm{~g}$ per day in males and $>7 \mathrm{~g}$ per day in females) using the self-monitoring device method should receive salt restriction education by registered dietitians or obtain precise values using the 24-h urine collection method. Despite its shortcomings, the use of a self-monitoring device is a convenient method of understanding the daily fluctuations in salt intake/excretion associated with dietary choices. Within the range of $6-9 \mathrm{~g}$ of salt excretion, the measurement errors of this method are relatively small (Figure 3). Thus, a self-monitoring device is a sufficient tool for evaluation of daily salt intake relative to Japanese standards $(8 \mathrm{~g}$ for males and $7 \mathrm{~g}$ for females). ${ }^{16,26}$

Moreover, the results of receiver operating characteristic curve analysis indicated that measuring $\mathrm{ON}$ salt is a sensitive test despite its low specificity; thus, for Japanese people, it may be a useful method for detecting individuals who are not meeting the daily salt-intake target of $<7$ g. ${ }^{26}$ Ohta et al. ${ }^{28}$ reported that subjects with higher 24 -h salt ( $>9.5 \mathrm{~g}$ in male and $>8 \mathrm{~g}$ in female) had significantly lower estimated glomerular filtration rates than those with lower 24-h salt excretion. Because the salt excretion values measured by a selfmonitoring device are relatively accurate within the range of 6-9g, management of salt intake using a self-monitoring device may be useful for motivating patients to reduce their salt intake and prevent renal dysfunction. In addition, because urinary salt excretion is a possible predictor of angiotensin receptor blocker resistance ${ }^{29}$ and excessive salt intake is a possible cause of resistant hypertension, ${ }^{30}$ daily self-monitoring of salt intake is very important. Our results indicate that the use of a self-monitoring device, which provides accurate results near the target levels of salt consumption, may be a useful motivational tool to assist individuals in reducing dietary salt intake. Future studies should investigate whether employment of a self-monitoring device leads to a decrease individual salt consumption.

There were several limitations to this study. The methods that we used may be unsuitable for larger-scale studies because simultaneous collection of $24 \mathrm{~h}$ and overnight urine over 3 days is difficult to complete and requires skill and health awareness on the part of the study subjects. However, although accurate simultaneous collection of $24 \mathrm{~h}$ and overnight urine over 3 test days is technically difficult and troublesome for participants, similar investigations should be performed using a larger number of participants in the future. Naturally, various technical errors occurred during sample collection by the subjects and the possibility that the low rate $(66 \%)$ of successful urine sample collection may have led to incorrect results cannot be denied. In addition, the daily salt intake of the subjects in this study was lower than that of an average Japanese individual, ${ }^{5}$ therefore, the results of this study may not be applicable to those with higher salt intakes or with larger daily fluctuations in salt intake. For example, the results may differ in older people or hypertensive patients. Another limitation of our study was that our observation period spanned only 3 days.
For a more precise evaluation, a longer observation period is needed. The influence of individual lifestyles, such as meal times and nocturia, ${ }^{31}$ and physiological characteristics, such as differences in urinary concentrating capacity between daytime and nighttime, were not examined in this study. We assessed whether urine collection was successful based on the predicted and actual 24-h-urinary creatinine excretion in this study; ${ }^{13,14,25}$ however, if the para-aminobenzoic acid check method ${ }^{32,33}$ had been used, the results may have been different.

In conclusion, estimation of 24-h salt excretion from overnight urine using a self-monitoring device allows recognition of daily fluctuations in urinary salt excretion. Thus, self-measured salt excretion using overnight urine samples is useful for estimating daily fluctuations in salt intake, and the self-monitoring device method may be an effective motivational tool for personal salt restriction.

\section{CONFLICT OF INTEREST}

The authors declare no conflict of interest.

\section{ACKNOWLEDGEMENTS}

We wish to thank Ms. Yuko Nakayama, Ms. Mariko Kuboyama, Ms. Yasue Miyai, and Ms. Fumiko Nakao for their excellent technical assistance. This work was supported by a Grant-in-Aid for Scientific Research, Japanese Society of the Promotion of Science (Grant No. 25750067).

1 Ikeda $\mathrm{N}$, Inoue $\mathrm{M}$, Iso $\mathrm{H}$, Ikeda S, Satoh $\mathrm{T}$, Noda M, Mizoue $\mathrm{T}$, Imano H, Saito $\mathrm{E}$, Katanoda K, Sobue T, Tsugane S, Naghavi M, Ezzati M, Shibuya K. Adult mortality attributable to preventable risk factors for non-communicable diseases and injuries in Japan: a comparative risk assessment. PLoS Med 2012; 9: e1001160.

2 Ando K, Kawarazaki H, Miura K, Matsuura H, Watanabe Y, Yoshita K, Kawamura M, Kusaka M, Kai H, Tsuchihashi T, Kawano Y. [Scientific statement] Report of the Salt Reduction Committee of the Japanese Society of Hypertension(1) Role of salt in hypertension and cardiovascular diseases. Hypertens Res 2013; 36: 1009-1019.

3 The INTERSALT Co-operative Research Group. Appendix tables. Centre-specific results by age and sex. J Hum Hypertens 1989; 3: 331-407.

4 Anderson CA, Appel LJ, Okuda N, Brown IJ, Chan Q, Zhao L, Ueshima H, Kesteloot H, Miura K, Curb JD, Yoshita K, Elliott P, Yamamoto ME, Stamler J. Dietary sources of sodium in China, Japan, the United Kingdom, and the United States, women and men aged 40 to 59 years: the INTERMAP study. J Am Diet Assoc 2010; 110: 736-745.

5 Asakura K, Uechi K, Sasaki Y, Masayasu S, Sasaki S. Estimation of sodium and potassium intakes assessed by two $24 \mathrm{~h}$ urine collections in healthy Japanese adults: a nationwide study. Br J Nutr 2014; 112: 1195-1205.

6 Aburto NJ, Ziolkovska A, Hooper L, Elliott P, Cappuccio FP, Meerpohl JJ. Effect of lower sodium intake on health: systematic review and meta-analyses. BMJ 2013; 346: $\mathrm{f} 1326$.

7 Miura K, Ando K, Tsuchihashi T, Yoshita K, Watanabe Y, Kawarazaki H, Matsuura H, Kusaka M, Kai H, Kawamura M, Kawano Y. [Scientific statement] Report of the Salt Reduction Committee of the Japanese Society of Hypertension(2) Goal and strategies of dietary salt reduction in the management of hypertension. Hypertens Res 2013; 36: 1020-1025.

8 de Vries H, Mesters I, Riet JV, Willems K, Reubsaet A. Motives of Belgian adolescents for using sunscreen: the role of action plans. Cancer Epidemiology. Cancer Epidemiol Biomarkers Prev 2006; 15: 1360-1366.

9 Okuda N, Nishi N, Ishikawa-Takata K, Yoshimura E, Horie S, Nakanishi T, Sato Y, Takimoto $\mathrm{H}$. Understanding of sodium content labeled on food packages by Japanese people. Hypertens Res 2014; 37: 467-471.

10 Murakami K, Sasaki S, Takahashi Y, Okubo H, Hirota N, Notsu A, Fukui M, Date C. Reproducibility and relative validity of dietary glycaemic index and load assessed with a self-administered diet-history questionnaire in Japanese adults. Br J Nutr 2008; 99: 639-648.

11 Fukumoto A, Asakura K, Murakami K, Sasaki S, Okubo H, Hirota N, Notsu A, Todoriki H, Miura A, Fukui M, Date C. Within- and between-individual variation in energy and nutrient intake in Japanese adults: effect of age and sex differences on group size and number of records required for adequate dietary assessment. J Epidemiol 2013; 23: 178-186.

12 Holbrook JT, Patterson KY, Bodner JE, Douglas LW, Veillon C, Kelsay JL, Mertz W, Smith JC Jr. Sodium and potassium intake and balance in adults consuming self-selected diets. Am J Clin Nutr 1984; 40: 786-793.

13 Ohta Y, Tsuchihashi T, Onaka U, Miyata E. Long-term compliance of salt restriction and blood pressure control status in hypertensive outpatients. Clin Exp Hypertens 2010; 32. 234-238. 
14 Ohta Y, Tsuchihashi T, Ueno M, Kajioka T, Onaka U, Tominaga M, Eto K. Relationship between the awareness of salt restriction and the actual salt intake in hypertensive patients. Hypertens Res 2004; 27: 243-246.

15 Liu K, Cooper R, McKeever J, McKeever P, Byington R, Soltero I, Stamler R, Gosch F, Stevens $\mathrm{E}$, Stamler J. Assessment of the association between habitual salt intake and high blood pressure: methodological problems. Am J Epidemiol 1979; 110: 219-226.

16 Sakaki M, Tsuchihashi T, Arakawa K, Fukui H, Kameda W, Tominaga M. Long-term variability of urinary salt excretion and blood pressure in hypertensive patients. Hypertens Res 2014; 37: 939-943.

17 Forster JL, Jeffery RW, VanNatta M, Pirie P. Hypertension prevention trial: do 24-h food records capture usual eating behavior in a dietary change study? Am J Clin Nutr 1990; 51: 253-257.

18 Tanaka T, Okamura T, Miura K, Kadowaki T, Ueshima H, Nakagawa $\mathrm{H}$, Hashimoto $\mathrm{T}$. A simple method to estimate populational 24-h urinary sodium and potassium excretion using a casual urine specimen. J Hum Hypertens 2002; 16: 97-103.

19 Kawasaki T, Itoh K, Uezono K, Sasaki H. A simple method for estimating 24 h urinary sodium and potassium excretion from second morning voiding urine specimen in adults. Clin Exp Pharmacol Physiol 1993; 20: 7-14.

20 Hirota S, Sadanaga T, Mitamura H, Fukuda K. Long-term compliance with salt restriction assessed using the spot urine method in Japanese cardiology outpatients. Hypertens Res 2013; 36: 1096-1099.

21 Kawano Y, Tsuchihashi T, Matsuura H, Ando K, Fujita T, Ueshima H. Report of the Working Group for Dietary Salt Reduction of the Japanese Society of Hypertension: (2) Assessment of salt intake in the management of hypertension. Hypertens Res 2007; 30: 887-893.

22 Yamasue K, Tochikubo O, Kono E, Maeda H. Self-monitoring of home blood pressure with estimation of daily salt intake using a new electrical device. J Hum Hypertens 2006; 20: 593-598.

23 Tsuchihashi T, Kai H, Kusaka M, Kawamura M, Matsuura H, Miura K, Ando K, Maruyama S, Hayabuchi H, Takagi Y, Nakahigashi N, Sato T, Kawano Y. [Scientific statement] Report of the Salt Reduction Committee of the Japanese Society of Hypertension (3) Assessment and application of salt intake in the management of hypertension. Hypertens Res 2013; 36: 1026-1031.
24 Tochikubo O, Uneda S, Kaneko Y. Simple portable device for sampling a whole day's urine and its application to hypertensive outpatients. Hypertension 1983; 5: 270-274.

25 Yasutake K, Horita N, Murata Y, Koyama S, Enjoji M, Tsuchihashi T. Estimated urinary salt excretion by a self-monitoring device is applicable to education of salt restriction. Hypertens Res 2015; 38: 143-148.

26 Ministry of Health La, Lobour and Welfare of Japan. Dietary Reference Intakes for Japanese, 2015. DAI-ICHI SHUPPAN Co: Tokyo, Japan. 2014 in Japanese.

27 Yasutake K, Sawano K, Shono N, Tsuchihashi T. Validation of a self-monitoring device for estimating 24-hour urinary salt excretion. Asia Pac J Cclin Nutr 2013; 22: 25-31.

28 Ohta Y, Tsuchihashi T, Kiyohara K, Oniki H. High salt intake promotes a decline in renal function in hypertensive patients: a 10-year observational study. Hypertens Res 2013; 36: $172-176$.

29 Hasegawa H, Kanozawa K, Asakura J, Takayanagi K, Komuro O, Fukada H, Tokushima H, Kogure $H$, Matsuzawa M, Mitarai T. Significance of estimated salt excretion as a possible predictor of the efficacy of concomitant angiotensin receptor blocker (ARB) and low-dose thiazide in patients with ARB resistance. Hypertens Res 2013; 36: 776-782.

30 Shimosawa T. Salt, the renin-angiotensin-aldosterone system and resistant hypertension. Hypertens Res 2013; 36: 657-660.

31 Yamori $Y$, Kihara M, Fujikawa J, Soh Y, Nara Y, Ohtaka M, Horie R, Tsunematsu T, Note S, Kukase M. Dietary risk factors of stroke and hypertension in Japan - Part 2: Validity of urinalysis for dietary salt and protein intakes under a field condition. Jpn Circ J 1982; 46: 939-943.

32 Knuiman JT, Hautvast JG, van der Heyden L, Geboers J, Joossens JV, Tornqvist H, Isaksson B, Pietinen P, Tuomilehto J, Poulsen L, Flynn A, Shortt C, Boing H, Yomtov B, Magathues E, Angelico F, Stetanutti C, Fazio S, Cantina R, Ricci G, Richopoulou A, Katapoti Z. A multi-centre study on completeness of urine collection in 11 European centres. I. Some problems with the use of creatinine and 4-aminobenzoic acid as markers of the completeness of collection. Hum Nutr Clin Nutr 1986; 40: 229-237.

33 Murakami K, Sasaki S, Takahashi Y, Uenishi K, Watanabe T, Kohri T, Yamasaki M, Watanabe R, Baba K, Shibata K, Takahashi T, Hayabuchi H, Ohki K, Suzuki J. Sensitivity and specificity of published strategies using urinary creatinine to identify incomplete 24-h urine collection. Nutrition 2008; 24: 16-22. 